\title{
The Laplace Microarchitecture for Tracking Data Uncertainty and Its Implementation in a RISC-V Processor
}

\author{
Vasileios Tsoutsouras \\ Signaloid / University of Cambridge \\ United Kingdom \\ Chatura Samarakoon \\ University of Cambridge \\ United Kingdom
}

\author{
Orestis Kaparounakis \\ Signaloid \\ United Kingdom \\ James Meech \\ University of Cambridge \\ United Kingdom
}

\author{
Bilgesu Bilgin \\ University of Cambridge \\ United Kingdom \\ Jan Heck \\ University of Cambridge \\ United Kingdom
}

\author{
Phillip Stanley-Marbell \\ Signaloid / University of Cambridge \\ United Kingdom
}

\begin{abstract}
We present Laplace, a microarchitecture for tracking machine representations of probability distributions paired with architectural state. We present two new methods for in-processor distribution representations which are approximations of probability distributions just as floating-point number representations are approximations of real-valued numbers. Laplace executes unmodified RISC-V binaries and can track uncertainty through them. We present two sets of ISA extensions to provide a mechanism to initialize distributional information in the microarchitecture and to allow applications to query statistics of the distributional information without exposing the uncertainty representations above the ISA.

We evaluate the accuracy and performance of Laplace using a suite of 21 benchmarks spanning domains ranging from variational quantum algorithms and sensor data processing to materials properties modeling. Monte Carlo simulation on the benchmarks requires $2076 \times$ more instructions on average (and up to $21343 \times$ in some cases) to achieve the same accuracy that Laplace can achieve in a single execution. Compared to state-of-the-art alternatives to Monte Carlo, Laplace achieves an average $1.3 \times$ accuracy improvement versus PaCAL [22] and more than 4.6× accuracy improvement versus the method used by the NIST Uncertainty Machine [26], quantified using the Wasserstein distance to Monte Carlo.

Unlike existing methods for uncertainty tracking which require software to be rewritten in a domain-specific language or extensive source-level changes, Laplace achieves all of these benefits while requiring no changes to existing binaries in order to track uncertainty through them, with only minimal changes required to get uncertainty information into the microarchitecture. We have deployed an implementation of Laplace as a commercial product in the form of a cloud-accessible virtual machine.
\end{abstract}

Permission to make digital or hard copies of part or all of this work for personal or classroom use is granted without fee provided that copies are not made or distributed for profit or commercial advantage and that copies bear this notice and the full citation on the first page. Copyrights for third-party components of this work must be honored.

For all other uses, contact the owner/author(s).

MICRO '21, October 18-22, 2021, Virtual Event, Greece

(c) 2021 Copyright held by the owner/author(s).

ACM ISBN 978-1-4503-8557-2/21/10.

https://doi.org/10.1145/3466752.3480131

\section{CCS CONCEPTS}

- Computer systems organization $\rightarrow$ Reduced instruction set computing; Embedded hardware; • Mathematics of computing $\rightarrow$ Probabilistic representations; • Hardware $\rightarrow$ Emerging architectures.

\section{KEYWORDS}

uncertainty tracking, distributional representations, arithmetic on distributions, RISC-V

\section{ACM Reference Format:}

Vasileios Tsoutsouras, Orestis Kaparounakis, Bilgesu Bilgin, Chatura Samarakoon, James Meech, Jan Heck, and Phillip Stanley-Marbell. 2021. The Laplace Microarchitecture for Tracking Data Uncertainty and Its Implementation in a RISC-V Processor. In MICRO-54: 54th Annual IEEE/ACM International Symposium on Microarchitecture (MICRO '21), October 18-22, 2021, Virtual Event, Greece. ACM, New York, NY, USA, 16 pages. https: //doi.org/10.1145/3466752.3480131

\section{INTRODUCTION}

Uncertain data are ubiquitous in computing systems. One common example is sensor measurements: there is always uncertainty, no matter how precise the instrument, between the recorded value (the measurement) and the quantity being measured (the measurand) [23]. This form of measurement uncertainty can be quantified by using multiple simultaneous measurements or by performing repeated measurements at a rate faster than the measurand can change. It is then possible to quantify the variation across measurements using statistical analysis or by noting the number of significant (i.e., unchanging) digits across the measurements. Such numerically-quantified uncertainty is referred to in the literature as aleatoric uncertainty [53]. Uncertainty may also exist when there is insufficient information about a quantity of interest. Such uncertainty in values resulting from incomplete information is often referred to in the literature as epistemic uncertainty.

Despite the relevance of uncertainty in computing systems, existing computer architectures neither have support for representing uncertainty, nor for arithmetic on uncertain values. Computer architectures today represent uncertain values with single particle values (i.e., data with no associated distribution), usually by taking the mean value as the representation for use in computation. In 


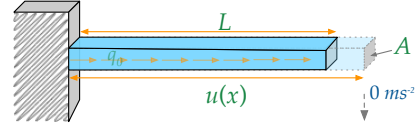

(a) Beam extends by $u(x)$ based on its (b) Uncertainty of beam extenstiffness $(E)$ and load $\left(q_{0}\right)$.

Figure 1: Uncertainty in stiffness (Young's modulus, $E$ ) leads to uncertainty in extension $u(x)$ for a given applied load $q_{0}$.

much the same way that the abstraction of floating-point number representations and floating-point arithmetic improved the ease of implementation of real-valued computations, architectural and microarchitectural support for representations of uncertainty could similarly enable new approaches to computation.

\subsection{Example uses of uncertainty tracking}

Figure 1(a) shows an engineering problem for which uncertainties in the problem parameters have real-world implications. The figure shows a beam of cross-sectional area $A$ and length $L$ made of material with Young's modulus $E$ [25], loaded with an applied axial load $q_{0}$ per unit length. The beam's extension $u(x)$ at position $x$ from the cantilever, is

$$
u(x)=\frac{q_{0}}{2 A E}\left(2 x L-x^{2}\right) .
$$

Because of material variabilities or variations in atmospheric conditions, the Young's modulus may be uncertain and this uncertainty can be quantified with a probability distribution for the Young's modulus rather than using a single number. In the same way that engineers will often want to evaluate a floating-point implementation of Equation 1 rather than one where the parameters are constrained to integer values, it is useful to evaluate Equation 1 with parameters such as the Young's modulus as distributions representing uncertainty. The implementations of analytic models such as Equation 1 or their finite-element counterparts may be in legacy or third-party libraries, making it attractive to have methods for tracking uncertainty that work on existing program binaries.

Figure 2 shows a second example of how uncertainty in the inputs to a computation can lead to uncertainty in the computation's result. The figure shows images from the MNIST dataset known to be ambiguous [35], along with distributions of labels for the images, assigned by 50 observers in a user study we conducted for this work. For example, for the first (left-most) image in the sequence ("Q"), Figure 2(a) shows the distribution of labels, with peaks at 0 and 6. Figure 2(i) shows the result of statistical sampling and propagation of these distributions for the digits $d_{i}$ through the expression $\sum_{i=0}^{7} d_{i} 10^{i}$ to determine the distribution of plausible interpretations of the handwritten 8-digit sequence "6405 C65 5 ".

The evaluation of uncertainty in Figure 1(b) and Figure 2(i) uses the standard (but expensive) method of statistical sampling, often referred to as the Monte Carlo method [40], with 30000 runs. In contrast to this expensive approach, Laplace's methods for tracking uncertainty make tracking uncertainty through arbitrary binaries as straightforward to achieve as implementing these computations using floating-point arithmetic. The results in Section 5 show that the methods for tracking uncertainty achieve accuracy close to that of the expensive Monte Carlo method.

\subsection{Laplace}

This article presents Laplace, a microarchitecture for tracking machine representations of probability distributions paired with architectural state. We present and evaluate two different machine-level representations of approximations of probability distributions. Just as fixed-point and floating-point number representations trade the number of bits in their representations for accuracy with respect to the represented real-valued number, the distribution representations we present trade the representation size for accuracy of representing empirical probability distributions. The representations allow computing systems to efficiently represent uncertain quantities even when those quantities have rarely-occurring highmoment values in the tails of the distribution.

Laplace honors traditional program semantics: Laplace allows programs to associate distributional information with registers and by extension all memory words that are used to load and store register values. In addition to maintaining the non-uncertain architectural state in registers, arithmetic instructions in Laplace use the distributional information in the microarchitecture associated with register operands to update the distributional information of the result register. Our microarchitectural implementation tracks distributional information across all operations in the floating-point datapath. In applications that do not ingest distributional information, the distributions are degenerate with point mass. In applications which use Laplace's extensions to set the distributions associated with data in a program's input or state, the microarchitecture tracks distributions across all operations in the floating-point datapath.

Laplace is ISA-independent: We implement the Laplace microarchitecture as the execution model for a commercial RISC-V virtual machine product implementing the RISC-V (RV32IMFD) ISA. In this implementation, Laplace represents and tracks uncertainty across computation transparently to the default semantics of the RISC-V ISA. Our implementation provides a mechanism to input uncertainty information into the processor and to query the uncertainty information which Laplace maintains in parallel with the non-uncertain architectural state, in the form of two groups of instruction extensions (Table 1) which together form a new RISC$\mathrm{V}$ "xua" extension. These extensions purposefully do not expose the uncertainty representations to applications. They only allow applications 10 to provide uncertainty distributions in a canonical form (as samples) and 2 to query derived properties (such as the $n$th moment) of the microarchitectural distributions. We extend GCC (version 8.2) and Binutils (version 2.32) to support these RISC$\mathrm{V}$ instruction extensions and we implement high-level language bindings to these extensions for $\mathrm{C} / \mathrm{C}++$ but our implementation can already, in principle, support any host language supported by GCC 8.2. We chose to use the 32-bit RISC-V ISA as a starting point for pragmatic implementation reasons but Laplace can in principle be implemented within any instruction set architecture.

Why implement uncertainty tracking below the ISA abstraction: Facilities for computing with uncertainty could be implemented in many ways: As a software-only implementation in a 

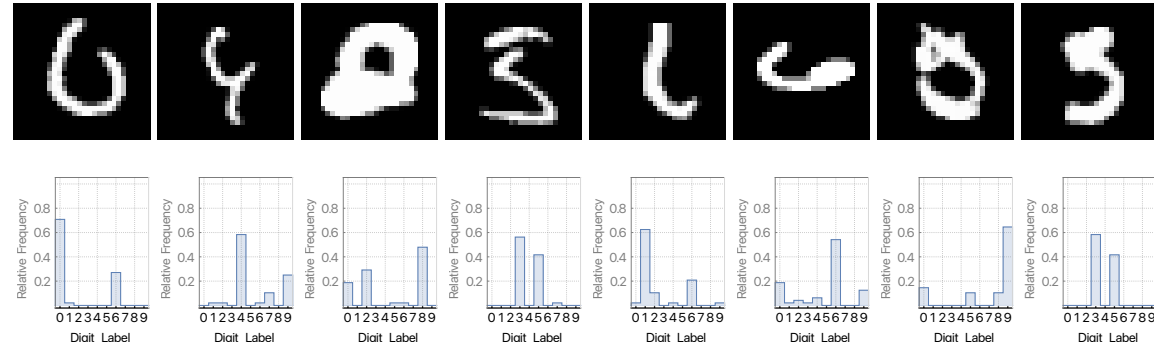

(b)

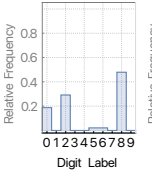

(c)

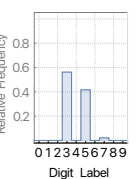

(d)

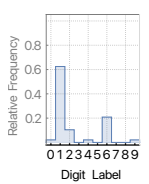

(e)

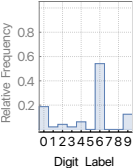

(f)

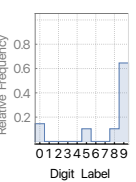

(g)

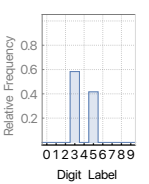

(h)

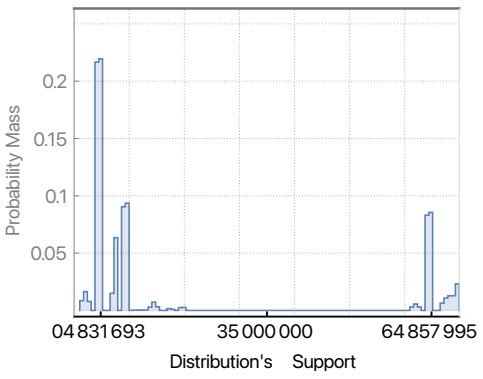

(i)

Figure 2: Eight ambiguous images [35] from the MNIST dataset and the distribution of labels (a-h) assigned to each by 50 observers. Based on these uncertainties in the labels, we can determine the distribution of plausible values of the 8-digit number using standard floating-point arithmetic instructions and can query the uncertainty associated with the result (i).

library [30], as a domain-specific language or new type system embedded in a host language [7], or below the ISA abstraction (whether in hardware or in a virtual machine). We chose a microarchitecturelevel abstraction because it is scalable to future improved machinelevel representations for non-parametric distributions beyond those we have developed to date. By tracking uncertainty below the ISA, Laplace can track distributional information through any staticallyor dynamically-linked library of the running application.

\subsection{Contributions}

Laplace makes five contributions to the state-of-the-art:

(1) New methods for distribution representation and arithmetic. We present two finite-dimensional representations for storing and operating on random variables (Section 2.2) and algorithms for performing arithmetic operations on these distribution representations in order to propagate the distributional information (Section 2.3).

(2) Laplace microarchitecture. We present Laplace, a microarchitecture for computing on data associated with distributional information (Section 3.1).

3 Insights from new tools to support uncertainty-tracking microarchitectures. We implement a complete compilation toolchain for the extensions we make to the RISC-V ISA to benefit from the Laplace microarchitecture, based on the GNU GCC compiler. We show how programs can exploit the benefits of Laplace with minimal changes to source code and with the possibility of tracking uncertainty through pre-compiled libraries.

4 Demonstration of new applications enabled by easilyaccessible uncertainty tracking. We evaluate Laplace using a suite of 21 benchmarks including examples of applications which benefit from uncertainty tracking, ranging from variational quantum algorithms and sensor data processing to materials properties modeling.

5 Comparison to the state of the art. We compare the accuracy and performance of Laplace to the standard reference of a Monte Carlo simulation. The results show that Laplace achieves the same accuracy as Monte Carlo simulations, executing $2076 \times$ fewer instructions on average and up to $21343 \times$ fewer instructions when using larger in-processor representations. Compared to state-of-the-art alternatives to Monte Carlo, Laplace achieves a $1.3 \times$ improvement in accuracy versus PaCAL [22] and more than $4.6 \times$ accuracy improvement compared to the method used by the NIST Uncertainty Machine [26]. We quantify accuracy improvement using the Wasserstein distance of the uncertainty tracking results compared to Monte Carlo results.

Laplace achieves all of these benefits while requiring no changes to binaries in order to track uncertainty through them, with only minimal changes required in order to get uncertainty information into the microarchitecture.

Laplace is in use commercially, as the execution model for a cloud-based virtual machine, targeted at domains including physical system modeling, autonomous systems, sensor data processing, machine learning, and quantum computing ${ }^{1}$.

\section{DISTRIBUTION REPRESENTATIONS}

We implement two complementary methods for representing uncertainty within the Laplace microarchitecture.

\subsection{Terminology}

The mean value or expected value of a random variable $X$ with probability mass function $f_{X}(x)$ is

$$
E(X)=\sum_{x: f_{X}(x)>0} x f_{X}(x)
$$

We often refer to the mean value of $X$ as $\mu_{X}$. Let $k$ be a positive integer. The $k$ th moment $m_{k}$ of $X$ is

$$
m_{k}=E\left(X^{k}\right) \text {. }
$$

The $k$ th centralized moment $\sigma_{k}$ of random variable $X$ is

$$
\sigma_{k}=E\left(\left(X-m_{1}\right)^{k}\right) \text {. }
$$

We refer to $\sigma_{2}$ as the variance, or $\operatorname{var}(X)$, of the random variable $X$. The variance provides a measure of how much $X$ deviates from its mean value.

Terminology in the context of Laplace: Laplace operates on machine representations of probability mass functions. In the same way that each value of a floating-point register is an approximate

\footnotetext{
${ }^{1}$ This instance of the Laplace microarchitecture is available at https://signaloid.io.
} 
representation of a real-valued number, each value in Laplace's uncertainty representation is an approximation of an entire probability mass function (not just of a probability, which is a real-valued number in $[0,1])$. The distributions that Laplace handles need not be nameable or parametric distributions such as uniform, Bernoulli, binomial, Gaussian, Laplacian, and so on. Laplace does not assume that the distributions in a computation are all the same, does not assume that the distributions involved in arithmetic operations are the same, and does not assume that a given item of machine state remains in the same distribution over time (i.e., Laplace does not assume homoscedasticity). Laplace does not expose its internal representation to applications above the ISA but instead allows applications to evaluate derived properties of distributions such as the expectation, centralized moments, and tail probability.

\subsection{Definitions of representations}

We introduce two microarchitecture-level representations of uncertainty distributions. Because the representations are of finite length, they are an approximation of the probability density function of the original random variable. The methods share an underlying representational primitive which we refer to as the Dirac mixture representation.

Dirac mixture representation: Let $\delta(x)$ be the Dirac delta function - a unit impulse at position $x$. Given a value $x_{0} \in \mathbb{R}$, we view $\delta\left(x-x_{0}\right)$ as a probability mass function and we call it a particle value (or just particle). Using this definition, we transform an array of particle values $x_{1}, \ldots, x_{M}$ into a probability mass function using a weighted sum:

$$
f_{X}(x)=\sum_{n=1}^{M} p_{n} \delta\left(x-x_{n}\right), \text { where } p_{n} \in[0,1], \sum_{n=1}^{M} p_{n}=1 .
$$

In-memory storage of Dirac mixtures: A Dirac mixture requires only a series of positions $\left(x_{i}\right)$ and probability masses $\left(p_{i}\right)$ for its description. For a random variable $d$, we represent its Dirac mixture of size $N$ as an array of $2 N$ floating-point values. For the $i$ th Dirac delta of the mixture, Laplace stores the position $\left(d_{\text {pos }}[i]\right)$ and probability mass $\left(d_{\text {mass }}[i]\right)$.

Regularly-quantized representation (RQHR): This representation is conceptually similar to histograms, which uniformly split the representational overhead across the support of the distribution. To create an RQHR from a set of empirical samples, we divide the support of the samples into $N$ bins of equal width $L$. We define the height of each bin $1 \leq i \leq N$ as $p_{i}$ and set it equal to the relative frequency of the source particles falling within the $i$ th bin. We store the RQHR as a Dirac mixture of size $N$, setting $d_{\text {pos }}[i]$ of the Dirac mixture equal to the median of the lower and upper bound of the bin and $d_{\text {mass }}[i]$ equal to $p_{i}$ of the $i$ th histogram bin of RQHR.

Telescoping torques representation (TTR): The telescoping torques representation (TTR) is based on the intuition that the mean value of a set of samples splits the samples into two sets with equal probability torque (i.e., the product between the sample value and its probability). This allows TTR to capture the effects of unlikely, yet high-value samples even at small representational sizes.

Let $\operatorname{src}_{\mathrm{DM}}$ be a Dirac mixture of particle values of samples from a random variable $X$. We convert $\operatorname{src}$ DM to a TTR of size $N>0$ using

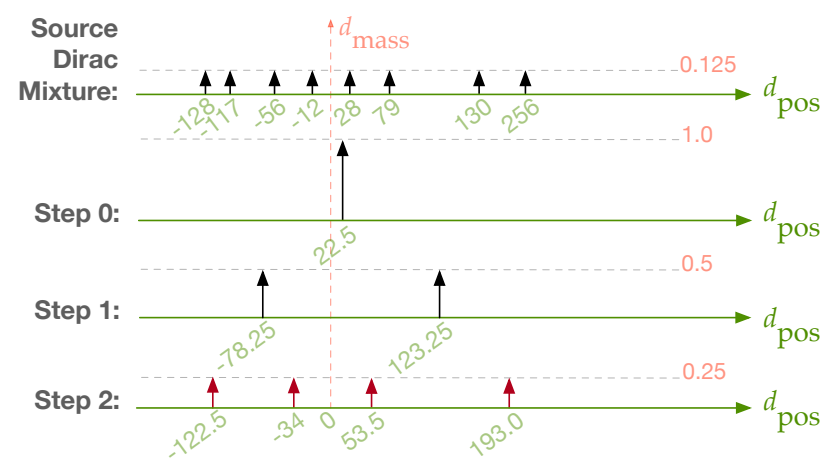

Figure 3: A Dirac delta (solid vertical arrow) placed at the mean of the Dirac mixture representation of $\operatorname{src}_{\mathrm{DM}}$ (top) is its $0^{\text {th }}$-order TTR (Step 0). In Step 1, the algorithm splits the source Dirac mixture using the mean $(\mu=22.5)$ of Step 0 and calculates the $1^{\text {st }}$-order TTR comprising two Dirac deltas. In Step 2, the algorithm uses the positions of these two Dirac deltas to subdivide the source Dirac mixture and calculates four Dirac deltas which form the $2^{\text {nd }}$-order TTR of $s r c_{\mathrm{DM}}$.

a recursive algorithm that takes $\log _{2} N$ steps. We call this number of steps the order of the TTR. At each step, the algorithm splits the input Dirac mixture on its mean value $\mu$, creating two Dirac mixtures which are the inputs of two new calls to the algorithm. Figure 3 shows an example for $N=4$, starting from a $s c_{\mathrm{DM}}$ of 8 particles. In step 0 , the method creates the $0^{\text {th }}$-order TTR of $s c_{\mathrm{DM}}$ which is a single Dirac delta located at the mean $(\mu)$ of $\operatorname{src}_{\mathrm{DM}}$ (i.e., $\left.d_{\text {pos }}[0]=22.5\right)$ with associated weight equal to the sum of the probability masses of all Dirac deltas in $\operatorname{src}_{\mathrm{DM}}\left(d_{\text {mass }}[0]=1.0\right)$.

Using $\mu$ as a boundary, step 1 splits $s c_{\mathrm{DM}}$ into two mixtures (each with four Dirac deltas in this example) and repeats the process for each mixture. The $1^{\text {st }}$-order TTR of $\operatorname{src} \mathrm{DM}$ is the union of the $0^{\text {th }}$ order TTRs of each sub-mixture. In the example of Figure 3, this corresponds to two Dirac deltas with probability mass equal to 0.5 , each one at the mean value of each sub-mixture. In step 2, the process repeats, further dividing the sub-mixtures of step 1 into two and calculating their $0^{\text {th }}$-order TTRs. This results in the $2^{\text {nd }}$-order TTR of $\operatorname{src}_{\mathrm{DM}}$, consisting of four Dirac deltas.

\subsection{Arithmetic operations on representations}

Let $\Phi$ be a function such that $\Phi: \mathbb{R}^{2} \rightarrow \mathbb{R}$ and let $X$ and $Y$ be two discrete random variables represented using our distributional representations. We define the necessary algorithms to compute the distributional representation of the discrete random variable $Z=\Phi(X, Y)$, for $\Phi$ as one of addition, multiplication, subtraction, and division. For RQHR and TTR, these basic arithmetic operations on uncorrelated random variables are circular convolutions of their positions and probability masses. Algorithm 1 shows the addition of two uncorrelated random variables represented with Dirac mixtures of size $N$. We store the results of the operations on the Dirac delta positions and masses of the two source representations in $d s t_{\mathrm{DM}}$, which is a Dirac mixture representation of size $N \times N$. We use the algorithms of Section 2.2 to convert $d s t_{\mathrm{DM}}$ to RQHR or TTR. 


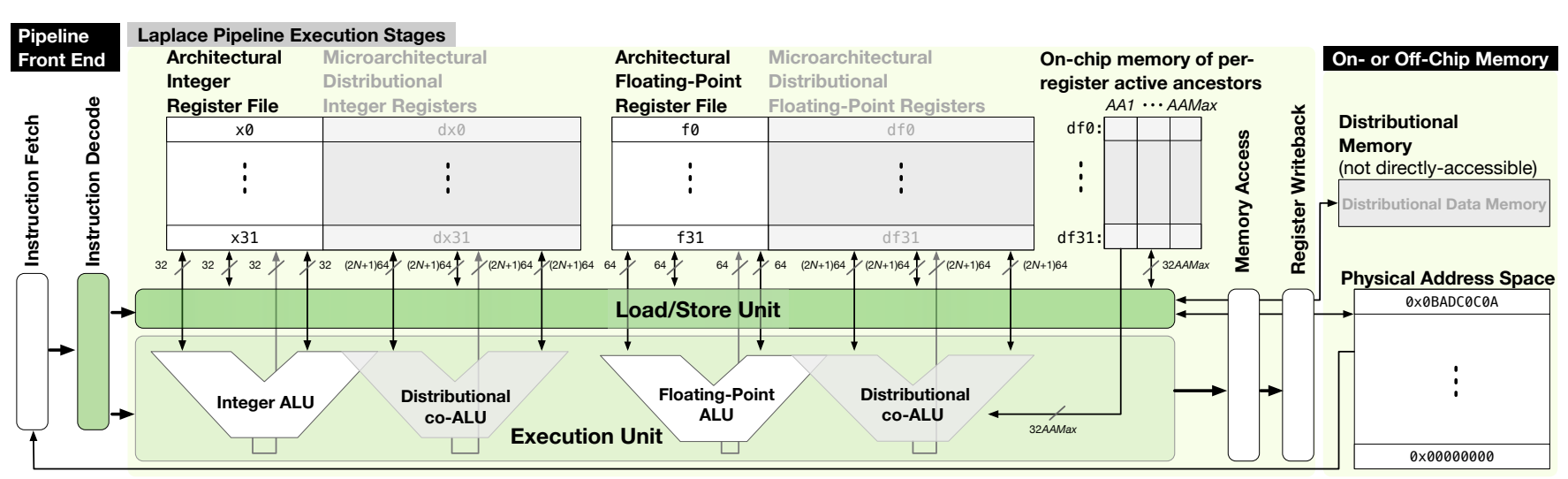

Figure 4: For each integer or floating-point ALU instruction, a distributional co-ALU performs the uncertainty tracking, fetching the uncertainty representations for the source operands from the corresponding distributional register file (not visible at the ISA level), performing the distributional arithmetic on the internal distribution representation of size $N$ (Section 2.2), and storing the result back in the distributional register file. An additional on-chip memory holds $A A M a x$ ancestor addresses for each distributional register necessary for autocorrelation tracking (Section 3.3). Color legend: Unmodified architectural state is in white ( $\square$ ); microarchitectural structures modified to allow applications to access derived information on uncertainty representations are in green $(\square)$; microarchitectural structures specific to uncertainty-distribution tracking (and not exposed above the ISA) are in grey ( ).

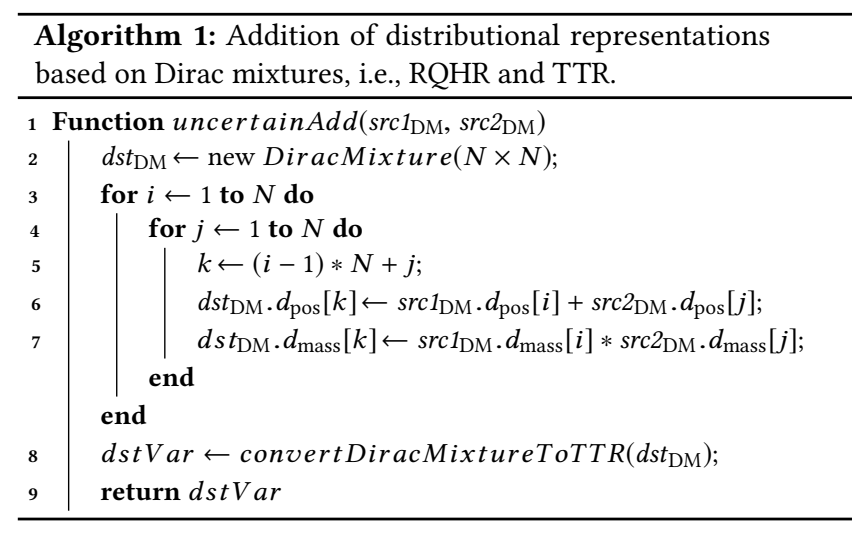

Both representations are of fixed size and do not increase in size as computation progresses.

To multiply two uncorrelated random variables, we replace line 6 of Algorithm 1 with a multiplication. Adding or multiplying a particle value by a Dirac mixture representation variable results in an offset or scaling of the Dirac deltas positions, respectively. We use this property to calculate the subtraction of two random variables. We implement division of two random variables of Dirac mixture representation as a multiplication of the dividend with the reciprocal of the divisor. We construct the latter by calculating the reciprocals of the positions of its Dirac mixture representation. The implementation which we evaluate in this work does not handle the case of arithmetic operations on random variables with arbitrary correlation. The implementation we evaluate only handles the case of autocorrelated arithmetic operations, i.e., expressions where a source operand appears multiple times. We elaborate on this in Section 3.3.

\subsection{Summary statistics on representations}

Let $X$ be a random variable represented as an RQHR or TTR of size $K$. Laplace can calculate the following statistical properties of $X$. Nth centralized moment: For any integer $N \geq 0$, the $N$ th moment of $X$ is given by $\sigma_{N}=E\left((X-E(X))^{N}\right)$ where $E(X)=$ $\sum_{i=0}^{K} d_{\text {pos }}[i] d_{\text {mass }}[i]$. Nth mode/anti-mode: The $N$ th mode of $X$ is the particle value $x$ at which the probability mass function $f_{X}$ takes its $N$ th highest value and is calculated as $d_{\text {pos }}\left[i_{N}\right]$ where $i_{N}$ is the index at which $d_{\text {mass }}$ takes the $N$ th highest value. $N$ th anti-mode is calculated similarly but with $i_{N}$ being the index at which $d_{\text {mass }}$ takes the $N$ th lowest value. If $N$ is greater than the size of the distributional representation the statistic evaluates to a NaN. Distribution's support minimum or maximum value: Returns the minimum or maximum value of the Dirac mixture positions of the distributional representation of $X$ (i.e., minimum or maximum of $d_{\text {pos }}$ ). Tail probability: Given a cutoff value $x_{0} \in \mathbb{R}$ we calculate the tail probability of $X$ as $\operatorname{Pr}\left(X>x_{0}\right)=\sum_{\forall i \in[0, K] \text { s.t. } d_{\text {pos }}[i]>x_{0}} d_{\text {mass }}[i]$. We calculate the probability $\operatorname{Pr}\left(X \leq x_{0}\right)$ as $1.0-\operatorname{Pr}\left(X>x_{0}\right)$.

\section{LAPLACE MICROARCHITECTURE}

Laplace tracks distributional information within the microarchitecture, transparently to applications. Each instance of the Laplace microarchitecture supports a single distributional representation (Section 2.2), e.g., RQHR of size 8 or TTR of size 4 , as a design-time choice. Implementing the Laplace microarchitecture does not introduce new data hazards and does not impact branch predictors and prefetchers of the architecture for which it is implemented (RISC-V RV32IMFD in this work).

\subsection{Register files and ALUs}

Figure 4 shows the microarchitectural structure of Laplace. Laplace does not modify the number of registers of the architecture within 


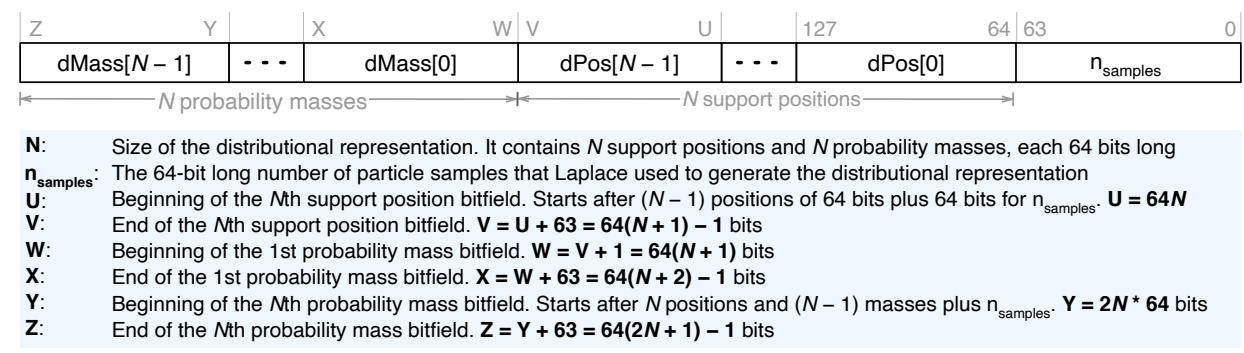

Figure 5: Bit-level representation of TTR/RQHR.

which it is implemented. Instead, it extends the integer and floatingpoint register files with additional microarchitecture-level distributional registers which store the bits of the distributional information that correspond to each register of the ISA-visible architecture. Figure 5 shows the bit-level representation of an RQHR or TTR of size $N$. The lower-order 64 bits store the number of particle samples used to derive the distributional representation. The next $N$ 64-bit values store the support positions of the representation. They are followed by the $N$ 64-bit values of the probability masses.

Let fo be a conventional floating-point register of the architecture within which we implement Laplace (in this work, RISC-V RV32IMFD) and let dfø be the respective microarchitecture-level distributional floating-point register. Laplace performs all arithmetic and logic instructions on both the conventional and distributional registers in parallel. For example, the addition of source registers $\mathrm{f} 1$ and $\mathrm{f} 2$ into destination register $\mathrm{fo}$ also triggers the addition of the distributional information of registers df1 and df2 into the distributional register $\mathrm{dfo}$. The semantics of non-distributional register values and operations on them remain unchanged. Tracking of distributional information happens in parallel to (and not affecting the behavior of) the non-distributional architectural state.

Laplace extends both integer and floating-point arithmetic and logic units (ALUs) with two distributional co-ALUs. The conventional, unchanged ALU operates on the particle values of the source registers. The distributional co-ALU performs the same operation on the distributional representations of the source registers using the algorithms of Section 2.3. The distributional co-ALU can calculate summary statistics (Section 2.4) of the distributional representation of a source register.

\subsection{Loading/storing uncertainty information}

A load instruction in Laplace loads the distributional representation that corresponds to an address of Laplace's main memory to the distributional part of the destination register. A store instruction stores the distributional information of the source register to the main memory of Laplace. Figure 4 shows how a portion of the physical memory of the processor implementing the Laplace microarchitecture stores the distributional representations. The load/store unit of Laplace maps an address accessed by an application to the address that stores its distributional representation.

Section 4 introduces an additional load instruction that initializes the distributional information of a destination register by creating a distribution representation from an in-memory array of source particle samples.
Figure 6 shows the implementation of the hardware module that converts source samples to TTR. The module comprises multiple levels of conversion units, one level for each step involved in the conversion of an array of samples in the form of a Dirac mixture to a TTR (Section 2.2). A pair of integers identifies each conversion unit. The first integer corresponds to the conversion step and the second is an increasing index, e.g., Conversion unit $[1,1]$ is the second conversion unit of conversion Step 1 (Section 2.2). Because Figure 6 shows an example for the conversion of input samples to TTR of size four, the output level consists of four conversion units which generate the four support positions (dmPos) and probability masses (dmMass) of the TTR of size four.

Each conversion unit has three inputs and three outputs. The first input is the memory address of the array of samples that the conversion unit will process. Laplace stores the samples in the main memory as a Dirac mixture, sorted by ascending support position (dmPos value). The second and third inputs are two integers that correspond to the starting index (startInd) and ending index (endInd) of the continuous array of samples that the conversion unit will process. Each conversion unit outputs a support position (dPos) value and a probability mass (dMass) value. The conversion unit calculates the output probability mass as dMass $=\sum_{\text {startInd }}^{\text {endInd }}$ dmMass $[i]$. The conversion unit calculates the mean value of the source Dirac mixture as $\mu=\sum_{\text {startInd }}^{\text {endInd }} \operatorname{dmPos}[i] * \mathrm{dmMass}[i]$ (Section 2.4). The conversion unit calculates the output support position as $d P o s=\frac{\mu}{d M a s s}$. The third output of the conversion unit is an integer kPartition which corresponds to the index below which all sorted support positions of the input Dirac mixture are less than the calculated dPos.

In intermediate conversion levels, the conversion units propagate their output kPartition to the next conversion level. Depending on the subset of arrays that they must process, the kPartition acts as the startInd or endInd value of the conversion units of the next level. In the final conversion level, the TTR conversion module writes the output distributional information to the distributional destination register and writes the mean value of the source samples to the conventional destination register.

\subsection{Correlation between register values}

Let $\mathrm{x}$ be a variable with distributional information. Section 2.3 introduced arithmetic operations on uncorrelated random variables. The correct calculation of expressions such as $x * x$ is a subset of the broader problem of handling correlated (i.e., non-independent) distributions. The correct execution of such autocorrelated arithmetic operations requires point-wise operations on the support of the 


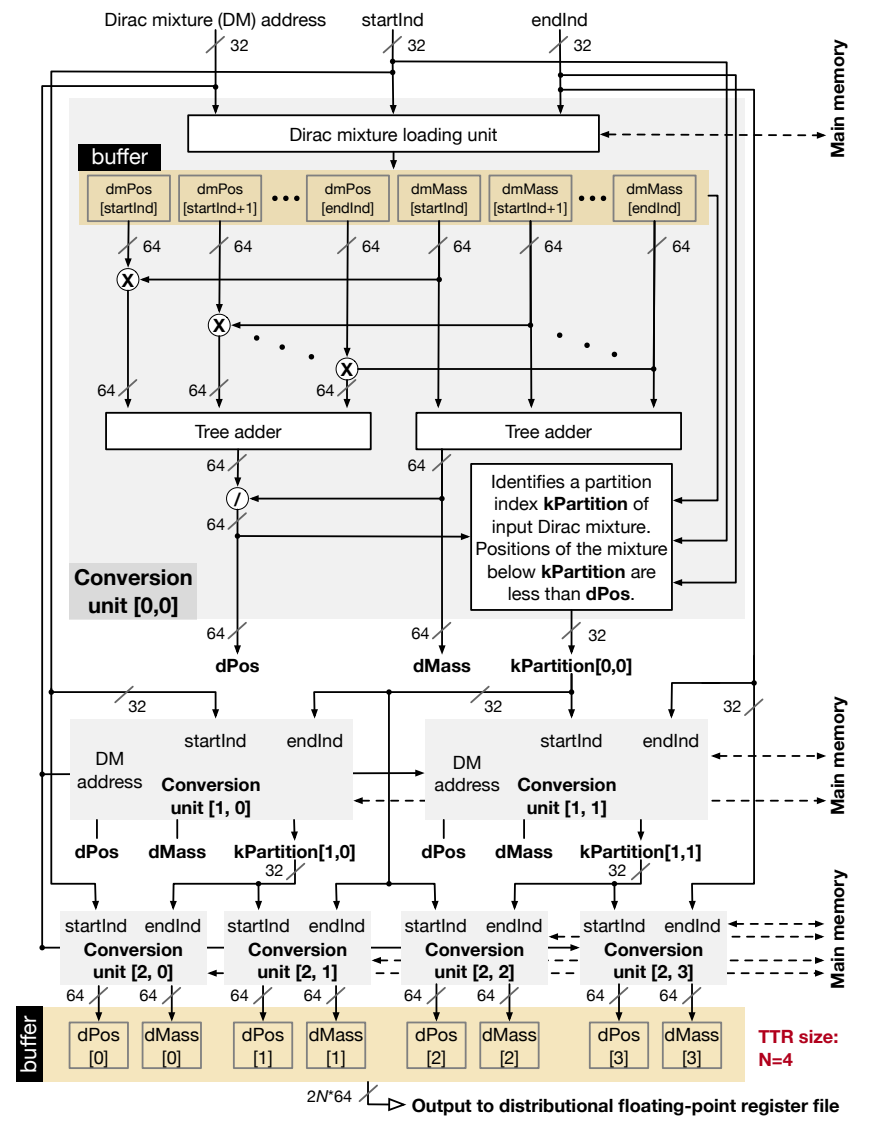

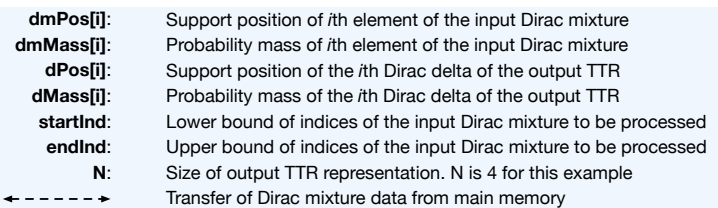

Figure 6: Module for conversion of an in-memory array of particle samples (stored as a Dirac mixture) to TTR of size $N$ ( $N$ is 4 for this example). The module comprises multiple levels of conversion units which take as input the address of the in-memory array of samples. Each unit processes the array indices dictated by startInd (lower bound) and endInd (upper bound) and calculates the support position (dPos) and probability mass (dMass) of the output TTR.

source operands. Figure 7 shows the calculation of $x * x$ with and without the correct handling of autocorrelation. Figure 7a shows the distribution of $\mathrm{x}$ created in Laplace using samples from a zero-mean Gaussian distribution. Figure $7 \mathrm{~b}$ shows the combined outcome of uncertainty tracking with autocorrelation tracking on or off and the ground truth of exhaustively evaluating $x * x$ for all samples of $x$ The evaluation of $x \star x$ when autocorrelation tracking is on is almost identical to the ground truth. Without such handling of arithmetic on autocorrelated random variables there are negative values in the support of the outcome distribution which is incorrect for the expression $\mathbf{x} * \mathbf{x}$.

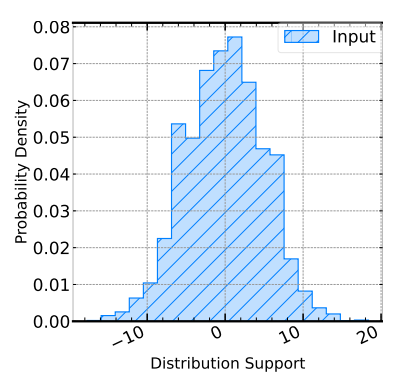

(a) $\times(N(0,5))$.

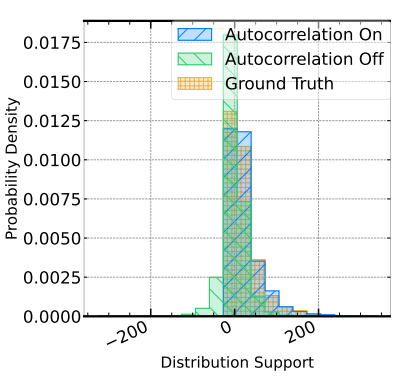

(b) Output $x * x$.
Figure 7: Input and output of expression $x * x$. Output plots show the result of uncertainty tracking (UT) with autocorrelation tracking on or off and the ground truth of exhaustively evaluating $x * x$ for all the samples of $x$. The evaluation of the expression with enabled autocorrelation tracking is almost identical to the ground truth. Evaluation without autocorrelation tracking leads to negative values in the support of the output distribution, which is incorrect.

Ancestor addresses tracking mechanism: Figure 8 shows the $C$ source code and RV32IFMD ISA disassembly of the calculation of the even powers of a variable $\mathrm{x}$, extracted from a function that uses Taylor series expansion to calculate the cosine of $\mathbf{x}$. The compiler uses two different registers ( $\mathrm{fa} 4$ and $\mathrm{fa5}$ ) to calculate $\mathrm{x} * \mathrm{x}$. Tracking only the identities of the source registers of instructions is not sufficient to track correlations between registers and by extension, variables with distributional information. The processor loads the values of both registers from the same memory address, which corresponds to the same variable of the source application. This is the core insight behind our autocorrelation tracking mechanism. The value of each floating-point register originates from one or more addresses of the main memory. By keeping track of these ancestor addresses we can dynamically identify correlations between any two registers of Laplace.

Each time Laplace executes a load instruction, it adds the source address to the set of ancestor addresses of the destination register rd. We use an on-chip memory of the Laplace microarchitecture to store a fixed number (AAMax) of ancestor addresses for each of the architectural registers. We evict addresses from this memory using a least-recently used (LRU) policy. When Laplace stores a register $r$ s to memory, its set of ancestor addresses is spilled to main memory. For each arithmetic operation, if the source registers have at least one common ancestor, Laplace executes the arithmetic operation in an autocorrelation-tracked manner. Laplace also updates the ancestor addresses of the destination register with the union of the ancestor addresses of the source registers.

Example of tracking correlations in Laplace: Figure 8 shows the execution of $x$ Power $*=x * x$ which involves two kinds of autocorrelated operations: (i) $x \star x$ and (ii) the more challenging case of $x$ Power * $(x * x)$ where Laplace needs to detect that $x$ Power is $x$ multiplied repeatedly with itself. Each row of Figure 8 corresponds to the execution of an assembly instruction (with increasing instruction index) and shows the set of ancestor addresses of the destination register before and after the execution of the instruction. 


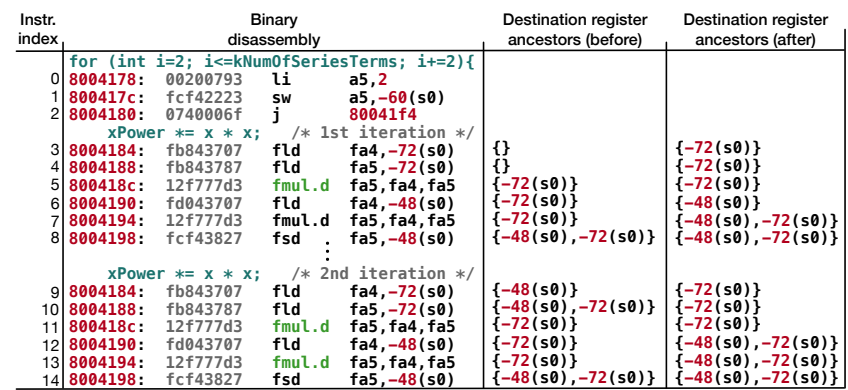

Figure 8: Excerpt from a RV32IMFD ISA binary that calculates the even powers of an input variable $x$. The compiler uses different registers for the calculation of $x * x$, which shows that we cannot rely on the register identities to identify correlations of the source registers of the arithmetic instructions. Laplace instead tracks the ancestor main memory addresses that have contributed to calculation of the value of each register. It performs autocorrelation-tracked execution of the fmul.d instructions shown in green (instruction indices 5, 11, and 13).

At instructions with indices 3 and 4, Laplace sets the ancestor addresses of registers fa4 and fa5 to $-72(\mathrm{~s} \theta)$, where $s 0$ is a saved integer register. Since fa4 and fa5 have identical ancestors, Laplace performs an autocorrelation-tracked multiplication at instruction $5(\mathrm{x} * \mathrm{x})$. At instruction 6, Laplace loads fa4 from an address that overwrites the ancestor addresses of fa4. At instruction 7 Laplace creates the set of ancestor addresses of destination register fa5 as the union of the ancestors of source registers fa4 and fa5. At instruction 8, Laplace stores the ancestors of fa4 in main memory. Laplace uses a microarchitectural structure, indexed by a main memory address to map the destination address $-48(s \theta)$ to the address of its ancestor set in main memory. During the second loop iteration, the instructions indexed 9-11 have the same effect as the respective instructions in the first loop iteration. At instruction 12, Laplace updates the ancestor set of fa4 using the ancestor set

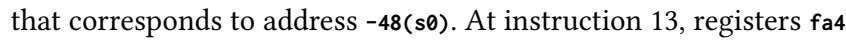
and fa5 share a common ancestor $(-72(s \theta))$. Laplace performs an autocorrelation-tracked multiplication which corresponds to xPower $*(x * x)$.

Distributional co-ALU: Figure 9 shows the internals of the distributional co-ALU. Using the information stored in the on-chip memory of active per-register ancestors (Figure 4) the co-ALU determines whether the source registers have common ancestor addresses in the autocorrelation detection unit. The unit sets the output signal srcoperandsAutocorrelationsignal if it detects autocorrelation. If not set, then the co-ALU executes Algorithm 1 (or a variant according to the instruction). The smaller ALU components perform the intended arithmetic operation on the support positions of the distributional source registers. For all arithmetic operations, the co-ALU multiplies the masses of the distributional source registers. An asserted srcoperandsAutocorrelationsignal signal disables non-pointwise units and the co-ALU performs a point-to-point operation on the source positions and masses. The buffered output

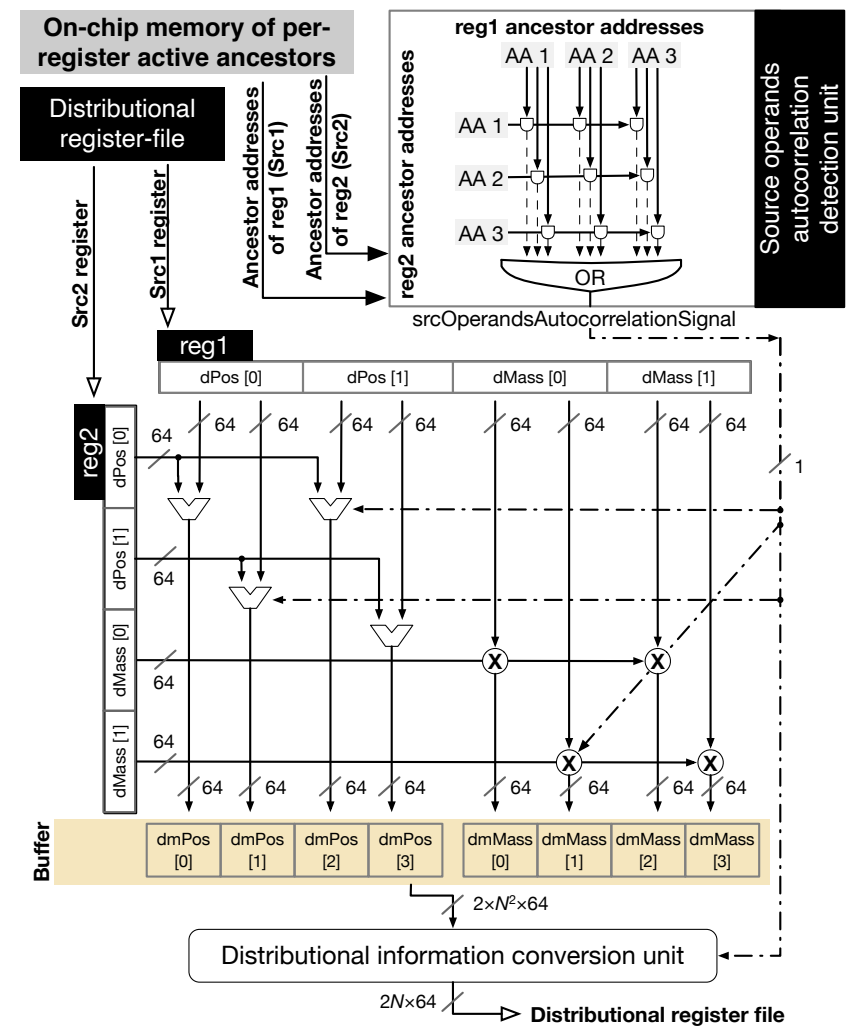

\footnotetext{
dPos[i]: Support position of the ith Dirac delta of the input register's TTR dMass[i]: Probability mass of the ith Dirac delta of the input register's TTR dmPos[i]: Support position of ith element of the output Dirac mixture dmMass[i]: Probability mass of $i$ th element of the output Dirac mixture N: Size of output TTR representation ( $\mathrm{N}$ is 2 in this example) Single bit signal of the equality of two ancestor addresses - - . Single bit signal controlling the execution of autocorrelated operations $\longrightarrow$ Access to the distributional register file (either integer of floating-point)
}

Figure 9: The distributional co-ALU takes as input two distributional source registers and their ancestor addresses. The autocorrelation detection unit exhaustively checks for common ancestors and sets signal srcoperandsAutocorrelationsignal, if any. The co-ALU executes Algorithm 1 (or the variant for the intended arithmetic operation). The srcoperandsAutocorrelationsignal controls which functional units of the coALU will operate on the source operands. A unit like that of Figure 6, converts the result Dirac mixture to TTR.

of both autocorrelation-tracked and uncorrelated-operand calculations is a Dirac mixture. A conversion unit like that of Figure 6, converts the Dirac mixture to TTR and forwards it to the distributional register file.

For the correct tracking of uncertainty in applications, Laplace needs to propagate distributional information through all floatingpoint operations. However, not all operations have the same distributional execution overhead. If one or both of the distributional source registers do not contain distributional data (i.e., are particle values) then the co-ALU performs a scaling of the support position or no operation, respectively. 


\section{INJECTING AND EXTRACTING UNCERTAINTY INFORMATION}

To initialize distributional information in Laplace and to allow applications to query statistics of the distributional information, we design and implement a new (i.e., non-standard) extension to the RISC-V instruction set: the xua RISC-V ISA extension ${ }^{2}$. The xua extension consists of nine new instructions whose only purpose is to provide a way to initialize (i.e., inject) or query (i.e., extract) uncertainty information without exposing the underlying microarchitecturelevel distribution representation to programs. These instructions are not required for tracking uncertainty in the Laplace microarchitecture. Table 1 shows the double-precision floating-point variants of our xua RISC-V ISA extension instructions. The single-precision floating-point variants follow the same design principles. In the following text, we show the instructions colored and unless specified, use floating-point registers.

Injecting distributional information into the microarchitecture: The xualdfs instruction creates a distribution representation from in-memory samples. Integer register $r s 1$ holds the address of the first of $N$ continuous values in the main memory of Laplace. Integer register rs2 holds $N$. The instruction stores the distribution representation into the distributional part of the microarchitectural (i.e, hidden) hardware structure associated with rd and the mean value of the input samples in the ISA-visible value of $r d$.

Representation-independent access of distributional information: Instructions xuanthmoment, xuanthmode, and xuanthantimode process the distribution representation associated with register $r \mathbf{1} 1$ and store its $N$ th moment, $N$ th mode, or $N$ th anti-mode in register rd, respectively. Integer source register rs2 stores $N$. Instructions xuasupportmin and xuasupportmax store the minimum and maximum value of the distribution representation support associated with rs 1 to $r d$. Instruction xuaprobabilitygt calculates the probability of the random variable with distributional information associated with rs1, being greater than the real value stored in rs2, and stores it in rd. Instruction xuasample samples the distributional information associated with register $r s 1$ and stores the particle sample in rd. For all xua* instructions of Table 1 we use the custom $\theta$ opcode reserved for custom instructions [51].

Mixture instruction: Instruction xuamixture (R4-type) takes as input two floating-point registers $r s 1$ and rs2, and a ratio particle value from register $r s 3$ and sets the distributional information of the register $r d$ as the mixture of the distributional information of $r s 1$ and $r s 2$ computed as $r s 3 * r s 1+(1-r s 3) * r s 2$. For this instruction we used the custom1 opcode [51].

Extension of the semantics of RV32IMFD ISA instructions: We extend the semantics of the fld instruction to load distribution representation data associated with an address of the main memory of Laplace into the microarchitecture's non-ISA-visible distribution registers. We extend the semantics of the fsd instruction to store the distribution data of the microarchitecture's non-ISAvisible distribution registers into the the main memory of Laplace.

For non-arithmetic floating-point instructions, such as conversion, sign-injection, and classi fy, Laplace appropriately propagates distributional information from source to destination registers. For

\footnotetext{
${ }^{2}$ The RISC-V instruction set manual [51] suggests the usage of " $\mathrm{X}$ " as the first letter of a non-standard ISA extension.
}

Table 1: Listing of the R-type instructions of the doubleprecision xua extension of the 32-bit RISC-V ISA. Laplace uses these instructions only to insert and query uncertainty. Uncertainty-tracking happens transparently without the need for any explicit instructions.

\begin{tabular}{cccccc|l}
\hline funct7 & rs2 & rs1 & funct3 & rd & opcode & Instruction \\
\hline 0011100 & rs2 & rs1 & 010 & rd & 0001011 & xualdfs.d \\
1111100 & rs2 & rs1 & 001 & rd & 0001011 & xuanthmoment.d \\
1111100 & rs2 & rs1 & 010 & rd & 0001011 & xuaprobabilitygt.d \\
1111100 & rs2 & $r s 1$ & 011 & rd & 0001011 & xuanthmode.d \\
1111100 & $r s 2$ & $r s 1$ & 100 & rd & 0001011 & xuanthantimode.d \\
1111100 & $r s 2$ & $r s 1$ & 101 & rd & 0001011 & xuasupportmin.d \\
1111100 & $r s 2$ & $r s 1$ & 110 & rd & 0001011 & xuasupportmax.d \\
1111100 & rs2 & rs1 & 111 & rd & 0001011 & xuasample.d \\
\hline
\end{tabular}

Table 2: Function definitions included in libUncertain library. The function names for double-precision values all have the prefix libUncertainDouble truncated to save space.

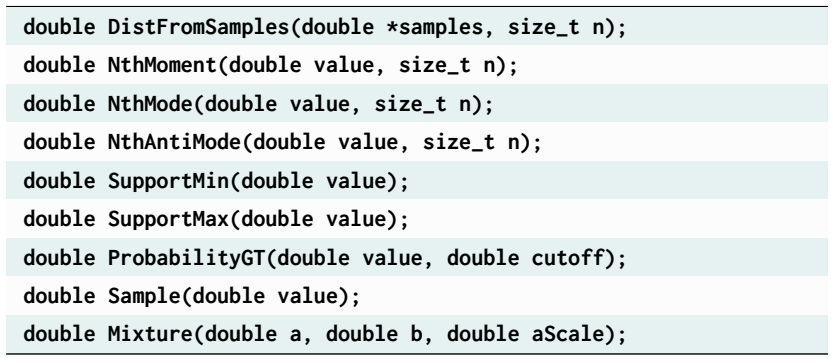

example, in the case of a negation using the fsgnj instruction, Laplace stores the distributional information that correspond to the ISA-visible source register to the distributional information that correspond to the ISA-visible destination register. Laplace then negates the support position values of the distributional information that correspond to the ISA-visible destination register. For compare instructions Laplace compares the particle values of the source registers to set the particle value of the destination register.

Control flow of binaries in Laplace: Binaries running on Laplace follow the conventional control flow. Applications can make a control flow decision based on distributional information by querying the distributional information of the registers of Laplace using the instructions of Table 1 .

Application development: We extend the GNU Compiler Collection (GCC) for application development. Our compilation infrastructure includes: (1) GCC 8.2.0 for the RV32IMFD ISA; (2) Binutils 2.32 for the RV32IMFD ISA, extended to support xua ISA extension; (3) Newlib 2.5.0.20170922 as the standard C library implementation; (4) Library libUncertain, which provides $C$ function wrappers for exposing the xua ISA extension instructions to applications.

Laplace treats any floating-point $C$ variable as a variable with distributional information, without any changes in the GCC compiler front-end. Developers interface with the xua ISA through the libUncertain library, which provides $\mathrm{C}$ function declarations that we implement using RISC-V xua ISA instructions. Table 2 summarizes the functions that operate on double-precision floating-point $\mathrm{C}$ variables. We also provide the respective single-precision functions. 


\section{EVALUATION}

We evaluate Laplace using a RISC-V microarchitectural emulator [45] modified to implement the Laplace microarchitecture and the xua RISC-V extension. Benchmarks use libUncertainDoubleDistFromsamples to initialize variables with distributional information from samples in data files. We use the mean of these samples as the input of the benchmark running on the conventional microarchitecture (an unmodified RV32IMFD RISC-V processor); the baseline of our accuracy and performance evaluations are repeated Monte Carlo iterations of the benchmarks running on this unmodified RV32IMFD RISC-V processor. Each run of the benchmarks uses point values sampled from a distribution (detailed further in Sections 5.1- 5.2). We execute Monte Carlo simulations of 30000 iterations and aggregate the results to obtain the output distribution. We refer to this as the Monte Carlo evaluation of the benchmarks.

Accuracy evaluation: Our accuracy metric is the Wasserstein distance [39] between the result of a single execution of the benchmark on Laplace using distributional inputs and the output of the Monte Carlo evaluation of the benchmark. To improve the interpretability of these distances, we normalize them by the Wasserstein distance between the conventional microarchitecture output and the Monte Carlo output. The normalized Wasserstein distance conveys the accuracy improvement factor of Laplace over the conventional microarchitecture. A value less than 1.0 means Laplace is more accurate than the conventional system.

Performance evaluation: To evaluate the performance improvement of Laplace compared to the Monte Carlo evaluation of the same benchmarks running over the baseline RV32IMFD RISC-V processor, we examine the number of ISA instructions that each method requires to achieve the same accuracy. For each benchmark, we calculate the number of Monte Carlo executions over the RV32IMFD RISC-V processor that are required to achieve the Wasserstein distance that Laplace achieves for each benchmark in a single execution.

Overview of benchmarks: Table 3 shows the benchmarks we used to evaluate Laplace. The benchmarks fall into three categories; (1) microbenchmarks that evaluate primitive arithmetic operations, (2) benchmarks from relevant works used for comparative evaluation, and (3) application benchmarks that evaluate the efficacy of Laplace in domains that deal with uncertainty. We used the $\mathrm{C}$ programming language to develop the benchmarks and compiled them for the RV32IMFDXua RISC-V ISA extension.

Results overview: Figure 10 shows the normalized Wasserstein distance that Laplace achieves for each benchmark, for each representation type (RQHR, TTR) and representation size (4 to 256). Figure 11 shows the dynamic instruction count improvement factor of Laplace over a Monte Carlo evaluation that achieves the same normalized Wasserstein distance (Section 5.4).

In general, RQHR leads to less accurate results, especially for small representation sizes, due to the use of uniform binning. By contrast, the ability of TTR to prioritize regions of high probability density makes it accurate in most of the benchmarks, even at small representation sizes. Across the 21 benchmarks, TTR achieves a $3.5 \times$ accuracy improvement on average compared to the conventional architecture, achieving more than $27 \times$ accuracy improvement in the quantum-avqe benchmark at a TTR size of 64. Laplace achieves these
Table 3: Overview of benchmarks evaluated on Laplace.

\begin{tabular}{l|l}
\hline Benchmark Name & Abbreviation \\
\hline (Microbenchmarks) & micro-add \\
Floating-point addition & micro-sub \\
Floating-point subtraction & micro-mul \\
Floating-point multiplication & micro-ac-mul \\
Floating-point multiplication with autocorrelation & micro-div \\
Floating-point division & micro-schlieren \\
(A+B)/(A-B) calculation & micro-matrix-mul \\
Floating-point matrix multiplication [30] & fpbench-daisy \\
Daisy from Fbench [4, 14] & fpbench-herbie \\
Herbie from FPbench [14, 36] & fpbench-real2float \\
FPTaylor-real2float from FPbench [14, 44] & \\
\hline (Comparisons) & pacal-cauchy \\
Product/quotient of Cauchy dist. (PaCAL [22]) & pacal-gp \\
Products of Gaussian distributions (PaCAL [22]) & pacal-tc \\
Thermal expansion coefficient (PaCAL [22]) & nistum-tc \\
Thermal expansion coefficient (NIST-UM [27]) & nistum-dv \\
Dynamic viscosity of solution (NIST-UM [27]) & \\
\hline (Applications) & gps-speed \\
Speed estimation using GPS data [7] & sensors-bme680 \\
Bosch BME680 conversion routines & mnist-number \\
MNIST [8] to positional number & brown-ham \\
Dislocation model from Brown and Ham [9] & fem-1d \\
Finite elements method 1D [10] & quantum-avqe \\
Accelerated variational quantum eigensolver [47] & \\
\hline
\end{tabular}

accuracy improvements with a single execution of the application whereas the Monte Carlo simulation requires 30000 executions.

\subsection{Microbenchmarks accuracy evaluation}

Arithmetic benchmarks: We use micro-add, micro-sub, micro-mul, and micro-div to assess the accuracy of Laplace for simple arithmetic operations. The inputs are samples from two Gaussian distributions with mean values 1.0 and 5.0 and variance of 1.0. For these simple arithmetic operations Laplace achieves an average accuracy improvement of $4.6 \times$ compared to the conventional architecture.

We use the expression $\frac{a+b}{a-b}$ (micro-schlieren) and a repeated multiplication (micro-ac-mul) to evaluate the effect of autocorrelation tracking in Laplace. The input samples for $a$ and $b$ follow Gaussian distributions with mean values 5.0 and -5.0 , respectively, and variance 10.0. Benchmark micro-ac-mul uses the same input as micro-mul. These benchmarks show average accuracy improvement of $1.08 \times$ (micro-schlieren) and 4.7× (micro-ac-mul), up to $98 \times$ for RQHR-256 in micro-ac-mul. We use micro-matrix-mul, a naive multiplication of two $8 \times 8$ matrices filled with uncertain data derived from a Gaussian distribution with mean value 1.0 and variance 1.0 , for an intensive assessment of the above properties. Laplace leads to improved accuracy of up to $87 \times$ for TTR-256.

FPBench: We use the FPbench [14] suite to evaluate Laplace on complicated floating-point arithmetic operations. Benchmarks fpbench-daisy [4], fpbench-herbie [36], and fpbench-real2float [44] contain numerical formulas for common mathematical functions, linear-algebraic operations, physics equations, and complex functions. For each one of the formulas we generate input samples by sampling a Gaussian distribution. To calculate the parameters of each source Gaussian distribution we take into account the input 


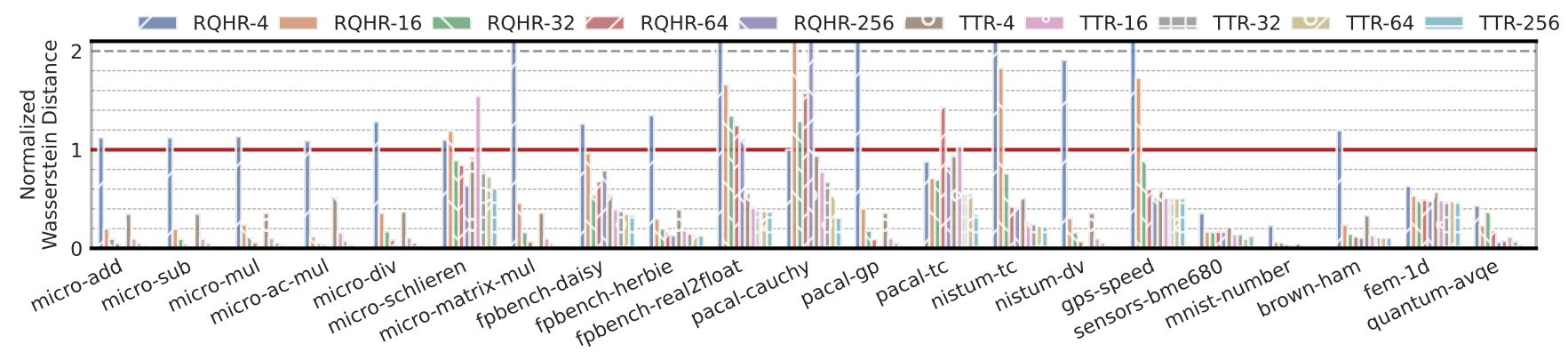

Figure 10: Laplace using TTR achieves up to $119 \times$ improvement in accuracy compared to the conventional system $(3.5 \times$ on average). The vertical axis gives the normalized Wasserstein distance, quantifying how close the result of using distributional representations with Laplace is, to the Monte Carlo output. A value less than $\mathbf{1 . 0}$ (below the red line) indicates Laplace is better than the conventional architecture. We used RQHR and TTR representations with sizes ranging from 4 to 256.

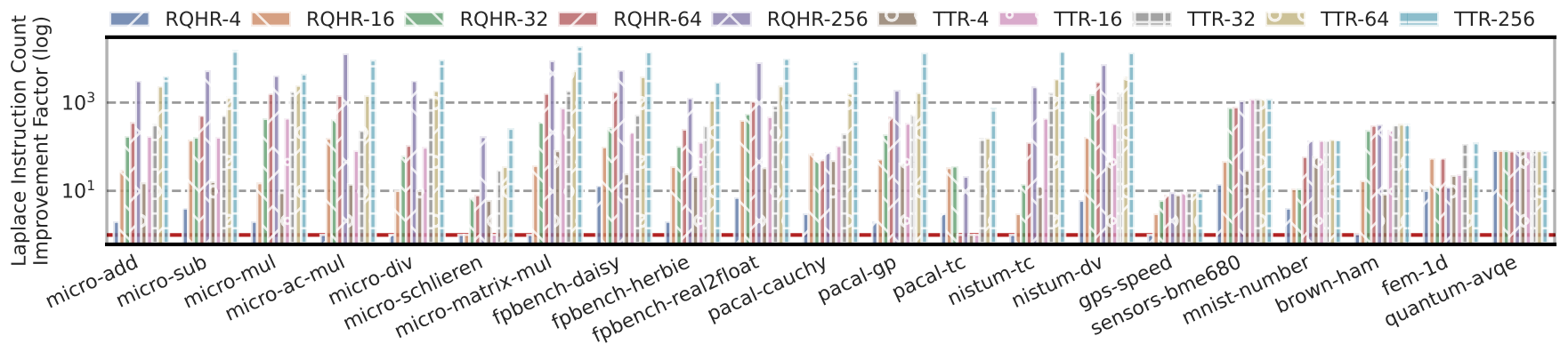

Figure 11: Dynamic instruction count improvement factor (vertical axis) of Laplace over a Monte Carlo simulation that achieves the same Wasserstein distance, for each benchmark and representation (horizontal axis). The red line at 1 indicates the baseline (no improvement). Laplace achieves average dynamic instruction count reduction of $2076 \times$ and a maximum reduction of $21343 \times$.

preconditions of each formula [14]. In daisy, Laplace with representation sizes greater than 16 achieves $1.5 \times$ (RQHR-64) to $2.5 \times$ (TTR-256) better accuracy compared to the baseline. In herbie and real2float, TTR achieves $2.4-5 \times$ better accuracy.

\subsection{Accuracy comparison to state of the art}

PaCAL [22]: We use three benchmarks from the PaCAL work: Cauchy distribution generation (pacal-cauchy), product of Gaussians (pacal-gp), and calculation of thermal expansion coefficient (pacaltc). We calculate the thermal expansion coefficient of a rod using its initial length $L_{a}$, final lengths $L_{b}$ and the temperature difference $\Delta T$. We assume that $L_{a}$ is uniformly distributed on $[9,10], L_{b}$ is uniformly distributed on $[11,12]$ and $\Delta T$ follows a Gaussian distribution with mean value 2.0 and variance 1.0. In pacal-cauchy, TTR manages up to $1.5 \times$ better Wasserstein distance, while RQHR offers no improvements. In pacal-gp RQHR and TTR show significant improvements over the baseline, averaging $2.8 \times$ accuracy improvement, up to more than $98 \times$ accuracy improvement for TTR-256. In pacal-tc RQHR shows $1.25 \times$ accuracy improvement on average, and TTR achieves on average $1.5 \times$ improved accuracy (up to $2.9 \times$ for TTR-256).
NIST Uncertainty Machine [27]: Using the NIST Uncertainty Machine web interface ${ }^{3}$, we execute two applications: calculation of thermal expansion coefficient and dynamic viscosity. We compare 1000000 Monte Carlo samples of the Uncertainty Machine output against the Laplace output. Thermal Expansion Coefficient: Like pacal-tc, but models the input parameters as Student's-t random variables [38] with 3 degrees of freedom [27]. Laplace achieves up to $4.6 \times$ better accuracy in this benchmark. Dynamic Viscosity: Calculates the dynamic viscosity of a sodium hydroxide solution in water by modeling the inputs as independent Gaussian variables [27]. Laplace achieves up to $119 \times$ better accuracy.

\subsection{Accuracy evaluation for complete applications}

Speed estimation using GPS data [7]: In human activity tracking, it is common to use GPS coordinates to estimate a person's movement speed. For this benchmark we use a public dataset of human activity with timestamped GPS coordinates and GPS accuracy values [21]. We adopt the methodology of Bornholt et al. [7] to derive a distribution of the GPS coordinates around a pair of GPS longitude and latitude values of the human activity dataset. We use the distributions of GPS coordinates to calculate the distribution of

\footnotetext{
${ }^{3}$ https://uncertainty.nist.gov/
} 


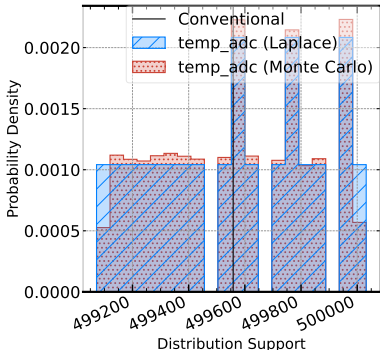

(a) ADC value (TTR-64)

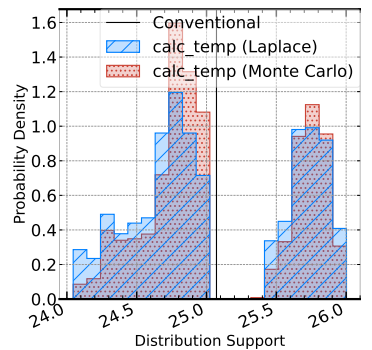

(b) Temperature (TTR-64)
Figure 12: Sampling the BME680 ADC values results in distributions even for small time intervals (Figure 12a). Laplace can track these distributions through the manufacturer's conversion routines and estimate the distribution of temperature (Figure 12b). The average estimate of the conventional architecture output falls in a zero-probability range.

the estimated speed between two GPS points (gps-speed). Compared to the conventional microarchitecture, the average improvement of the TTR in terms of Wasserstein distance from the Monte Carlo evaluation output is $1.9 \times$.

Bosch BME680 sensor conversion routines: The BME680 by Bosch is a temperature, pressure, and humidity sensor. Bosch provides routines for converting raw ADC values to meaningful measurements using 20 sensor-specific calibration constants. The sensors-bme680 benchmark evaluates the effect of noise in the ADC measurements and uncertainty in the calibration constants on the calibrated temperature, pressure, and humidity outputs of official commercial calibration firmware code provided by Bosch [43]. Figure 12a shows the noisy temperature ADC measurements and Figure $12 \mathrm{~b}$ their effect on the converted temperature. In this application, aleatoric uncertainty leads to a bimodal distribution of the output result. The conventional architecture output falls in a zeroprobability range, meaning that average-filtering the noisy ADC leads to a wrong result. Compared to the conventional microarchitecture, Laplace achieves on average $5.7 \times$ (up to $10 \times$ with TTR-64) smaller Wasserstein distance from the Monte Carlo output.

MNIST to positional number: Section 1.1 describes the mnistnumber benchmark which converts 8-digit sequences of MNIST images to plausible interpretations as decimal numbers. Figure 2(a) shows the distribution of labels assigned by 50 observers in a user study we conducted for this work. Laplace's accuracy improvement compared to the conventional microarchitecture averages $17.6 \times$ (up to $41 \times$ with TTR-256).

Brown-Ham dislocation model: This benchmark calculates the cutting stress of an alloy precipitate using the Brown-Ham dislocation model [1,9]. Anderson et al. [1] provide empirical value ranges for the inputs of the dislocation model. We assume that the inputs of the dislocation model follow a uniform distribution across these ranges. On average, in comparison to the conventional microarchitecture Laplace achieves $3.83 \times$ (up to $9.3 \times$ ) higher accuracy with respect to the Monte Carlo simulation.

One-dimensional finite element model: Section $1.1 \mathrm{de}-$ scribes an one-dimensional finite element model to calculate the

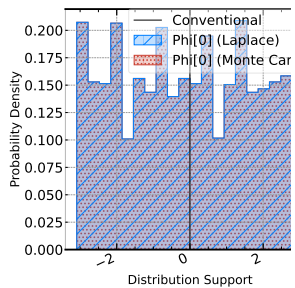

(a) Phi (Iteration 0)

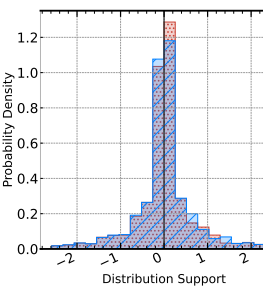

(b) Phi (Iteration 2)

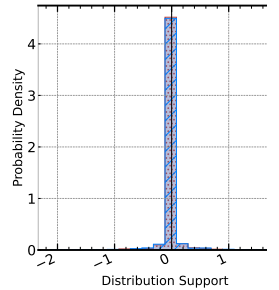

(c) Phi (Iteration 5)
Figure 13: Laplace is able to accelerate the QPE optimization of AVQE without using sampling to find better estimates of the posterior distribution of $\phi$ (using TTR-64).

extension of a beam when the model parameters have epistemic uncertainty. Benchmark fem-1d implements that model. We assume a uniform distribution of the Young's modulus input parameter of the benchmark and fixed particle values for the rest of the input parameters. Laplace achieves an average accuracy improvement of $2 \times$ compared to the conventional microarchitecture.

Accelerated variational quantum eigensolver: This quantum algorithm calculates ground states of a quantum system Hamiltonian, $H[13,47]$. The quantum-avqe benchmark tries to find the quantum state $\psi(k)$ that minimizes $\langle\psi(k)|H| \psi(k)\rangle$. Typically, this uses rejection sampling to calculate $P(\phi \mid E)$ from measurements of $P(E \mid \phi)$ from a quantum phase estimation (QPE) circuit [52]. With Laplace we can explicitly calculate the posterior from $P(E \mid \phi)$ and calculate $P(\phi)$. Over multiple iterations on Laplace, the algorithm can calculate better estimates of $P(\phi \mid E)$. Figure 13a shows the input of the quantum-avqe benchmark which we generate using samples from a uniform distribution. Figures $13 b$ and $13 c$ show the decrease in the posterior variance $P(\phi)$ after two and five iterations of the benchmark, respectively. Laplace achieves an accuracy improvement of $6.23 \times$ compared to the conventional microarchitecture (up to $41.3 \times$ with TTR-256).

\subsection{Performance improvement over Monte Carlo}

Figure 11 shows the dynamic instruction count ratio between the Monte Carlo and Laplace for all examined representation types and sizes. Except for RQHR-4, Laplace performs at least one order of magnitude better. On average, the accuracy that Laplace achieves in a single execution requires $2076 \times$ fewer instructions compared to the number of instructions that repeated Monte Carlo simulations require to achieve the same accuracy. The number of instructions that Laplace requires for the execution of a benchmark is independent of the size of the distributional representation. As the representation size increases, the accuracy of a single execution of Laplace increases and a greater number of Monte Carlo iterations are necessary to achieve the same level of accuracy. Thus, at higher representation sizes Laplace achieves even higher performance compared to Monte Carlo simulations, reaching up to $21343 \times$ improvement. 
Table 4: Cardinality of the largest set of ancestor addresses required by Laplace to evaluate each benchmark of Table 3 .

\begin{tabular}{llll}
\hline Benchmark & Ancestors & Benchmark & Ancestors \\
\hline micro-add & 0 & pacal-tc & 9 \\
micro-sub & 0 & nistum-tc & 4 \\
micro-mul & 0 & nistum-dv & 6 \\
micro-ac-mul & 5 & gps-speed & 5 \\
micro-div & 0 & sensors-bme680 & 25 \\
micro-schlieren & 6 & mnist-number & 2 \\
fpbench-daisy & 14 & brown-ham-model & 4 \\
fpbench-herbie & 4 & fem-1d & 23 \\
fpbench-real2float & 13 & quantum-avqe & 5 \\
pacal-cauchy & 9 & matrix-multiplication & 20 \\
pacal-gp & 9 & & \\
\hline
\end{tabular}

\subsection{Overhead of autocorrelation tracking}

To quantify the hardware overhead of our correlation tracking mechanism we measure the largest set of ancestor addresses that Laplace stores during the execution of each benchmark. Table 4 shows that the maximum number of required ancestors per benchmark is bounded and unaffected by the type or the size of the distributional representation. Thus, an on-chip memory of 32 ancestors per architectural register is large enough for all the benchmarks.

\section{RELATED RESEARCH}

Existing software approaches for representation and arithmetic operations of random variables frequently rely on Monte Carlo or first-order approximations. The NIST Uncertainty Machine [27] is a software tool for math operations on random variables that follow parametric distributions. It uses the first two centralized moments of the distribution [27] or Monte Carlo simulations. Manousakis et al. [30] represent random variables using their first two moments (mean value and variance) and their covariances. They use first-order Differential Analysis to approximate continuous and differentiable non-linear operations and use Monte Carlo simulation as fallback. PaCAL [22] carries out arithmetic operations on independent real-valued random variables by approximating their probability density functions with Chebyshev polynomials. This limits the density classes it can track through computation. In contrast to Laplace, PaCAL cannot handle empirical distributions.

Uncertain $<T>[6,7]$, a framework that represents random variables using user-specified sampling functions, and ProbZelus [3], a language for reactive probabilistic programming, use Bayesian networks to reduce the amount of dynamically-evaluated expressions, an optimization that Laplace does not implement yet. To the best of our knowledge, Laplace is the first microarchitecture for uncertainty tracking below the ISA abstraction. This allows applications to track and reason about uncertainty using unmodified programming languages and binaries.

Other hardware-based approaches adapted fuzzy logic [54] into microprocessor designs [12, 34, 41, 42, 48-50]. The fundamental limitation of this approach is that it uses only Bernoulli variables [18, $\S 4]$, and, thus, is not suitable for the types of problems that arise when dealing with uncertain data $[5, \S 2.4]$.
Attempts in approximate computing, like Truffle [16], Parrot [17], Green [2], and Flikker [29], introduce uncertainty to the computation, by using approximations, to gain performance or to save energy. Laplace does not aim to approximate input data or the outcome of computations. Laplace introduces new microarchitectural units for robust and accurate uncertainty tracking.

Particle filters $[15,28]$ track uncertainty but are more computeintensive than Laplace due to much larger uncertainty representations. Analogous to the concept of our microarchitectural approach, previous work has investigated accelerating particle filters in the microarchitecture $[19,20,37]$ or with SIMD operations [11, 3133]. The unscented transformation [24, 46] achieves uncertainty tracking with low resource usage and can in principle be an alternative technique for representing and tracking uncertainty inside the Laplace microarchitecture.

\section{CONCLUSIONS}

This article presents Laplace, a microarchitecture for tracking uncertainty using machine representations of probability distributions, and two new methods for such representations. Laplace uses these methods for compact, in-processor storage of distributional information as well as propagation of distributional information through arithmetic operations. We show how the Laplace RISC-V ISA extension, xua, allows programs to inject uncertainty information into a microarchitecture and to extract it, without the microarchitecture revealing the underlying microarchitectural distribution representation. We present an overview of the Laplace microarchitecture and show the necessary microarchitectural components for Laplace to achieve uncertainty tracking below the ISA boundary. These include modules for initializing distributional representations using samples stored in the main memory of Laplace, units that track correlations between representations of random variables, and units that perform arithmetic operations on distribution representations.

We implement Laplace as the microarchitecture for a RISC-V RV32IMFD processor and evaluate its performance using a suite of 21 benchmarks. The results lead to two important insights. First, using the Monte Carlo simulation as the golden standard for uncertainty tracking, Laplace improves the uncertainty tracking accuracy of conventional computing systems and outperforms relevant stateof-the-art software methods. Second, the accuracy of uncertainty tracking that Laplace achieves in a single run requires more than three orders of magnitude fewer instructions compared to the instructions that Monte Carlo simulation needs to achieve the same level of accuracy. These insights validate designing uncertainty tracking as a microarchitecture feature, abstracted through conventional ISA semantics. Challenging research directions open up, including improving the in-processor distribution representations, correlation tracking between random variables in computation, and efficient hardware implementations of Laplace.

\section{ACKNOWLEDGMENTS}

This research is supported by an Alan Turing Institute award TU/B/000096 under EPSRC grant EP/N510129/1 and by EPSRC grant EP/V047507/1. 
Table 5: Directory path of each benchmark in the provided artifact, relative to artifacts-benchmarks-source-code/src/.

\begin{tabular}{|c|c|}
\hline Benchmark Abbreviation & Benchmark subdirectory \\
\hline micro-add & micro-benchmarks/double-add/ \\
\hline micro-sub & micro-benchmarks/double-subtract/ \\
\hline micro-mul & micro-benchmarks/double-multiply/ \\
\hline micro-ac-mul & micro-benchmarks/double-multiply-autocorrelation/ \\
\hline micro-div & micro-benchmarks/double-divide/ \\
\hline micro-schlieren & micro-benchmarks/schlieren/ \\
\hline micro-matrix-mul & linear-algebra/matrix-multiplication/ \\
\hline fpbench-daisy & numerical-methods/FPbench-benchmarks/daisy/ \\
\hline fpbench-herbie & numerical-methods/FPbench-benchmarks/herbie/ \\
\hline fpbench-real2float & numerical-methods/FPbench-benchmarks/fptaylor-real2float/ \\
\hline pacal-cauchy & comparative-evaluations/UPP-vs-PaCal/ \\
\hline pacal-gp & comparative-evaluations/UPP-vs-PaCal/ \\
\hline pacal-tc & comparative-evaluations/UPP-vs-PaCal/ \\
\hline nistum-tc & comparative-evaluations/UPP-vs-NISTUM/benchmark/thermal-expansion-coefficient/ \\
\hline nistum-dv & comparative-evaluations/UPP-vs-NISTUM/benchmark/dynamic-viscosity-solution/ \\
\hline gps-speed & comparative-evaluations/Speed-Estimation-using-GPS-data/ \\
\hline sensors-bme 680 & sensor-interfacing/BME680-conversion-routines/ \\
\hline mnist-number & numerical-methods/MNISTtoPositionalNumber/ \\
\hline brown-ham & numerical-methods/Brown-and-Ham-materials-precipitate-cutting-dislocation-model/ \\
\hline fem-1d & numerical-methods/FEM1D/ \\
\hline quantum-avqe & quantum-computing/accelerated-variational-quantum-eigensolver/ \\
\hline
\end{tabular}

\section{A ARTIFACT APPENDIX}

We provide the source code of our evaluated benchmarks as publicly available artifacts in Zenodo. For the artifact evaluation we provided a remote virtual machine with all necessary software dependencies, including the Laplace SDK, where reviewers reproduced the evaluation results of Section 5. The provided artifact includes scripts that enabled the reviewers to automate the evaluation of benchmarks. The cloud-accessible version of the Laplace microarchitecture is available at https://signaloid.io.

\section{A.1 Artifact check-list (meta-information)}

- Program: We provide the source code for all the benchmarks detailed in Section 5. Their compressed size is $29.1 \mathrm{MB}$ and their uncompressed size is $61 \mathrm{MB}$.

- Compilation: We make use of (1) the GCC compiler 8.2.0 for the RV32IMFD ISA; (2) Binutils 2.32 for the RV32IMFD ISA, extended to support xua ISA extension; (3) Newlib 2.5.0.20170922 as the standard $\mathrm{C}$ library implementation. We provided this cross-compilation infrastructure as part of the provided remote machine for the evaluation of artifacts.

- Binary: We do not provide the binaries of the examined benchmarks. Reviewers were able to build all benchmark binaries using the cross-compilation infrastructure on the remote machine that we provided. The artifacts include a script that automated the building and evaluation of all benchmarks (exec-MICR0-2021-ArtifactEvaluation-benchmarks.sh).

- Data set: For each benchmark we have created different input datasets. We provide these datasets as part of the archived benchmarks source code. Each benchmark directory contains an inputs/ directory where we store the input dataset of the benchmark.

- Hardware: We do not require any specific hardware.
- Run-time state: The Laplace emulator creates a standard initial state for the execution of our benchmarks. In general, the evaluation of our artifacts is not sensitive to run-time state.

- Execution: There are no specific execution conditions for the execution of our benchmarks. The evaluation of all our benchmarks for tracking of distributional information requires approximately one hour. The execution time of the Monte Carlo evaluation of our benchmarks depends on the user-defined number of Monte Carlo iterations. With the default number of Monte Carlo iterations in the provided execution scripts the execution takes 10-12 days.

- Output: Each execution of a benchmark on the Laplace emulator can generate two kinds of output: (1) The standard output of the benchmark. This can be the outcome of standard C standard library calls, e.g., printf. It can also be the output of the distributional representation of a variable using libuncertainDoublePrint. This library prints the distributional representation of its source variable in stdout. This print includes a vertical listing of the support positions and probability masses of the source variable with distributional information. It also contains a graphical representation of the probability masses magnitude; (2) The emulator can use the DWARF debugging information of its input binary to trace the evolution of values of a benchmark source code expression during the execution of the benchmark.

- Metrics: The Laplace emulator stores multiple pieces of information about the execution of a benchmark. Relevant to the evaluation of Section 5 are (i) the number of dynamic instruction which were executed for the evaluation of a benchmark (both for uncertainty tracking and Monte Carlo simulation) and (ii) the maximum number of ancestors stored for any register during the execution of an experiment.

- Amount of time needed to prepare workflow (approximately): The time needed by the artifact evaluators to prepare 
the workflow was low because we provided access to a remote machine that included all the necessary software dependencies.

- Publicly available and archived: Our artifacts are publicly available in Zenodo at https://doi.org/10.5281/zenodo.5150148.

- Code licenses: We provide our source code under the MIT license.

- Data licenses: We provide our input benchmark data under the MIT license.

- Workflow framework used: We do not use a workflow framework. We provide bash scripts that automate the evaluation process

\section{A.2 Artifact description}

At the top directory level (artifacts-benchmarks-source-code) the artifact contains two directories: scripts/ and src/. The scripts/ directory contains the scripts that automated the Laplace evaluation process for the reviewers. Under the src/ directory we provide the $\mathrm{C}$ source code of the benchmarks that we detail in Table 3. Table 5 shows the path to each benchmark relative to the artifactsbenchmarks-source-code/src/ directory. Each one of these subdirectories contains two directories: benchmark/ and inputs/. The benchmark/ directory contains the $\mathrm{C}$ source code of the respective benchmark. It also includes a Makefile for building and running the benchmark, as well as Laplace SDK configuration files ( $m$ files). The inputs/ directory contains the input files of the respective benchmark. These inputs contain artificially-generated or empirical samples that we use to load the distributional representations for benchmarks running over the Laplace SDK. We randomly sample these inputs when we run the Monte Carlo simulation of each benchmark.

\section{REFERENCES}

[1] M.J. Anderson, F. Schulz, Y. Lu, H.S. Kitaguchi, P. Bowen, C. Argyrakis, and H.C. Basoalto. 2020. On the modelling of precipitation kinetics in a turbine disc nickel based superalloy. Acta Materialia 191 (2020), 81-100. https://doi.org/10.1016/j. actamat.2020.03.058

[2] Woongki Baek and Trishul M Chilimbi. 2010. Green: A framework for supporting energy-conscious programming using controlled approximation. In Proceedings of the 31st ACM SIGPLAN Conference on Programming Language Design and Implementation. 198-209.

[3] Guillaume Baudart, Louis Mandel, Eric Atkinson, Benjamin Sherman, Marc Pouzet, and Michael Carbin. 2020. Reactive probabilistic programming. In Proceedings of the 41st ACM SIGPLAN Conference on Programming Language Design and Implementation. 898-912.

[4] Heiko Becker, Pavel Panchekha, Eva Darulova, and Zachary Tatlock. 2018. Combining tools for optimization and analysis of floating-point computations. In International Symposium on Formal Methods. Springer, 355-363.

[5] James Bornholt. 2013. Abstractions and techniques for programming with uncertain data. Master's thesis. Honors thesis, Australian National University.

[6] James Bornholt, Todd Mytkowicz, and Kathryn S McKinley. 2014. Uncertain< $\mathrm{T}>\mathrm{a}$ first-order type for uncertain data. In Proceedings of the 19th international conference on Architectural support for programming languages and operating systems. 51-66.

[7] James Bornholt, Todd Mytkowicz, and Kathryn S McKinley. 2015. Uncertain< $\mathrm{t}>$ : Abstractions for uncertain hardware and software. IEEE Micro 35, 3 (2015), $132-143$.

[8] Léon Bottou, Corinna Cortes, John S Denker, Harris Drucker, Isabelle Guyon, Larry D Jackel, Yann LeCun, Urs A Muller, Edward Sackinger, Patrice Simard, et al. 1994. Comparison of classifier methods: a case study in handwritten digit recognition. In Proceedings of the 12th IAPR International Conference on Pattern Recognition, Vol. 3-Conference C: Signal Processing (Cat. No. 94CH3440-5), Vol. 2. IEEE, 77-82.

[9] LM Brown and RK Ham. 1971. Dislocation-particle interactions. Strengthening methods in crystals (1971), 9-135.

[10] John Burkardt. 2019. https://people.sc.fsu.edu/ jburkardt/c_src/fem1d/fem1d. html

[11] Fredrik Bagge Carlson. 2020. MonteCarloMeasurements. jl: Nonlinear propagation of arbitrary multivariate distributions by means of method overloading. arXiv preprint arXiv:2001.07625 (2020).

[12] Alessandra Costa, Alessandro De Gloria, Fabrizio Giudici, and Mauro Olivieri. 1997. Fuzzy logic microcontroller. IEEE Micro 17, 1 (1997), 66-74.
[13] James R Cruise, Neil I Gillespie, and Brendan Reid. 2020. Practical Quantum Computing: The value of local computation. arXiv preprint arXiv:2009.08513 (2020).

[14] Nasrine Damouche, Matthieu Martel, Pavel Panchekha, Jason Qiu, Alex SanchezStern, and Zachary Tatlock. 2016. Toward a Standard Benchmark Format and Suite for Floating-Point Analysis. (2016).

[15] Pierre Del Moral. 1997. Nonlinear filtering: Interacting particle resolution. Comptes Rendus de l'Académie des Sciences-Series I-Mathematics 325, 6 (1997), 653-658.

[16] Hadi Esmaeilzadeh, Adrian Sampson, Luis Ceze, and Doug Burger. 2012. Architecture support for disciplined approximate programming. In Proceedings of the seventeenth international conference on Architectural Support for Programming Languages and Operating Systems. 301-312.

[17] Hadi Esmaeilzadeh, Adrian Sampson, Luis Ceze, and Doug Burger. 2012. Neural acceleration for general-purpose approximate programs. In 2012 45th Annual IEEE/ACM International Symposium on Microarchitecture. IEEE, 449-460.

[18] Merran Evans, Nicholas Hastings, and Brian Peacock. 2013. Statistical distributions (2 ed.). John Wiley \& Sons.

[19] Qifeng Gan, JMP Langlois, and Y Savaria. 2013. Parallel array histogram architecture for embedded implementations. Electronics letters 49, 2 (2013), 99-101.

[20] Qifeng Gan, JM Pierre Langlois, and Yvon Savaria. 2013. A reformulated systematic resampling algorithm for particle filters and its parallel implementation in an application-specific instruction-set processor. In 2013 IEEE 56th International Midwest Symposium on Circuits and Systems (MWSCAS). IEEE, 1415-1418.

[21] Daniel Garcia-Gonzalez, Daniel Rivero, Enrique Fernandez-Blanco, and Miguel R Luaces. 2020. A Public Domain Dataset for Real-Life Human Activity Recognition Using Smartphone Sensors. Sensors 20, 8 (2020), 2200.

[22] Szymon Jaroszewicz and Marcin Korzeń. 2012. Arithmetic operations on independent random variables: a numerical approach. SIAM fournal on Scientific Computing 34, 3 (2012), A1241-A1265.

[23] Joint Committee for Guides in Metrology, Working Group 1. 2008. Evaluation of measurement data-Guide to the expression of uncertainty in measurement. Int. Organ. Stand. Geneva ISBN 50 (2008), 134.

[24] Simon J Julier. 2002. The scaled unscented transformation. In Proceedings of the 2002 American Control Conference (IEEE Cat. No. CH37301), Vol. 6. IEEE, 45554559.

[25] Jeffrey R. Keaton. 2018. Young's Modulus. Springer International Publishing, Cham, 955-956. https://doi.org/10.1007/978-3-319-73568-9_298

[26] Thomas Lafarge and Antonio Possolo. 2015. The NIST Uncertainty Machine. NCSLI Measure 10, 3 (2015), 20-27. https://doi.org/10.1080/19315775.2015.11721732 arXiv:https://doi.org/10.1080/19315775.2015.11721732

[27] Thomas Lafarge and Antonio Possolo. 2015. NIST Uncertainty Machine-User's Manual. National Institute of Standards and Technology, Gaithersburg (2015).

[28] Jun S Liu and Rong Chen. 1998. Sequential Monte Carlo methods for dynamic systems. Fournal of the American statistical association 93, 443 (1998), 1032-1044.

[29] Song Liu, Karthik Pattabiraman, Thomas Moscibroda, and Benjamin G Zorn. 2011. Flikker: Saving DRAM refresh-power through critical data partitioning. In Proceedings of the sixteenth international conference on Architectural support for programming languages and operating systems. 213-224.

[30] Ioannis Manousakis, Îñigo Goiri, Ricardo Bianchini, Sandro Rigo, and Thu D Nguyen. 2018. Uncertainty propagation in data processing systems. In Proceedings of the ACM Symposium on Cloud Computing. 95-106.

[31] Simon Maskell, Ben Alun-Jones, and Malcolm Macleod. 2006. A single instruction multiple data particle filter. In 2006 IEEE Nonlinear Statistical Signal Processing Workshop. IEEE, 51-54.

[32] Henry Medeiros, Germán Holguín, Paul J Shin, and Johnny Park. 2010. A parallel histogram-based particle filter for object tracking on SIMD-based smart cameras. Computer Vision and Image Understanding 114, 11 (2010), 1264-1272.

[33] Henry Medeiros, Johnny Park, and Avinash Kak. 2008. A parallel color-based particle filter for object tracking. In 2008 IEEE Computer Society Conference on Computer Vision and Pattern Recognition Workshops. IEEE, 1-8.

[34] Asim M Murshid, Sajad A Loan, Shuja A Abbasi, and Abdul Rahman M Alamoud. 2011. VLSI Architecture of Fuzzy Logic Hardware Implementation: a Review. International fournal of Fuzzy Systems 13, 2 (2011).

[35] Curtis G. Northcutt, Lu Jiang, and Isaac L. Chuang. 2019. Confident Learning: Estimating Uncertainty in Dataset Labels. arXiv:stat.ML/1911.00068

[36] Pavel Panchekha, Alex Sanchez-Stern, James R Wilcox, and Zachary Tatlock. 2015. Automatically improving accuracy for floating point expressions. ACM SIGPLAN Notices 50, 6 (2015), 1-11.

[37] A Pasciaroni, JA Rodríguez, F Masson, P Julián, and E Nebot. 2014. VLSI architecture design for particle filtering in real-time. In 2014 Argentine Conference on Micro-Nanoelectronics, Technology and Applications (EAMTA). IEEE, 70-76.

[38] Karl Pearson. 1895. X. Contributions to the mathematical theory of evolution.-II. Skew variation in homogeneous material. Philosophical Transactions of the Royal Society of London.(A.) 186 (1895), 343-414.

[39] Aaditya Ramdas, Nicolás García Trillos, and Marco Cuturi. 2017. On wasserstein two-sample testing and related families of nonparametric tests. Entropy 19, 2 
(2017), 47.

[40] Reuven Y Rubinstein and Dirk P Kroese. 2016. Simulation and the Monte Carlo method. Vol. 10. John Wiley \& Sons.

[41] Valentina Salapura. 2000. A fuzzy RISC processor. IEEE Transactions on fuzzy systems 8,6 (2000), 781-790.

[42] Mamoru Sasaki and Fumio Ueno. 1994. 7.5 MFLIPS fuzzy microprocessor using SIMD and logic-in-memory structure. IEICE transactions on electronics 77, 7 (1994), 1075-1082.

[43] Bosch Sensortec. [n. d.]. BME680 sensor API. [Online]. Available: https: //github.com/BoschSensortec/BME680_driver, Accessed: 07/09/2021.

[44] Alexey Solovyev, Marek S Baranowski, Ian Briggs, Charles Jacobsen, Zvonimir Rakamarić, and Ganesh Gopalakrishnan. 2018. Rigorous estimation of floatingpoint round-off errors with symbolic taylor expansions. ACM Transactions on Programming Languages and Systems (TOPLAS) 41, 1 (2018), 1-39.

[45] Phillip Stanley-Marbell and Diana Marculescu. 2007. Sunflower: Full-system, Embedded, Microarchitecture Evaluation. In Proceedings of the 2nd International Conference on High Performance Embedded Architectures and Compilers (HiPEAC'07) Springer-Verlag, Berlin, Heidelberg, 168-182.

[46] Eric A Wan and Rudolph Van Der Merwe. 2000. The unscented Kalman filter for nonlinear estimation. In Proceedings of the IEEE 2000 Adaptive Systems for Signal Processing, Communications, and Control Symposium (Cat. No. 00EX373) Ieee, 153-158.
[47] Daochen Wang, Oscar Higgott, and Stephen Brierley. 2019. Accelerated Variational Quantum Eigensolver. Phys. Rev. Lett. 122 (Apr 2019), 140504. Issue 14. https://doi.org/10.1103/PhysRevLett.122.140504

[48] Hiroyuki Watanabe. 1992. RISC approach to design of fuzzy processor architecture. In [1992 Proceedings] IEEE International Conference on Fuzzy Systems. IEEE, 431-441.

[49] Hiroyuki Watanabe and David Chen. 1993. Evaluation of fuzzy instructions in a RISC processor. In [Proceedings 1993] Second IEEE International Conference on Fuzzy Systems. IEEE, 521-526.

[50] Hiroyuki Watanabe, David Chen, and Srinivas Konuri. 1996. Evaluation of $\mathrm{min} / \mathrm{max}$ instructions for fuzzy information processing. IEEE transactions on fuzzy systems 4, 3 (1996), 369-374.

[51] Andrew Waterman, Yunsup Lee, David A Patterson, and Krste Asanovic. 2014. The risc-v instruction set manual. volume 1: User-level ISA. California University Berkeley Dept of Electrical Engineering and Computer Sciences, Berkeley, CA 94720-1776 and 94720-1770.

[52] Nathan Wiebe and Chris Granade. 2016. Efficient Bayesian Phase Estimation. Phys. Rev. Lett. 117 (Jun 2016), 010503. Issue 1. https://doi.org/10.1103/ PhysRevLett.117.010503

[53] Robin Willink. 2013. Measurement uncertainty and probability. Cambridge University Press.

[54] Lotfi A Zadeh. 1996. Fuzzy sets. In Fuzzy sets, fuzzy logic, and fuzzy systems: selected papers by Lotfi A Zadeh. World Scientific, 394-432. 